\title{
A 4-Hz Fundamental Linewidth on-chip Microlaser
}

\author{
Lan Yang, Tao Lu, Tal Carmon, Bumki Min and Kerry J. Vahala \\ Caltech, 1200 E. California BLVD. Pasadena CA 91125. \\ lan@caltech.edu,vahala@caltech.edu,www.vahala.caltech.edu
}

\begin{abstract}
A compact laser source on a silicon chip with Shawlow-Townes linewidth (i.e., fundamental, quantum limited) down to a few Hertz is demonstrated in this work. The fundamental linewidth is observed to decrease with inverse optical power.

(C) 2007 Optical Society of America

OCIS codes: (120.3940) Metrology; (140.3410) Laser Resonators.
\end{abstract}

The development of stable and ultra-narrow linewidth laser source sees many applications in coherent communication systems, biosensing devices as well as high-resolution spectroscopy [1,2]. Jiang et. al. have reported a single-frequency fiber laser with record linewidth of $200 \mathrm{~Hz}$ [3]. In this work, we demonstrate the first micron-scale on-chip laser having a fundamental linewidth as narrow as $4 \mathrm{~Hz}$. The fundamental linewidth of a laser is given by the Schawlow-Townes formula,

$$
\Delta v=\frac{h v^{3} n_{s p}}{4 \pi} \cdot \frac{1}{P} \cdot \frac{1}{Q^{2}}
$$

where $\mathrm{Q}$ is the "cold cavity" quality factor of the laser cavity, $\mathrm{P}$ is output power, $v$ is the optical frequency and $\mathrm{n}_{\mathrm{sp}}$ is the spontaneous emission factor. Therefore, increasing the cavity $\mathrm{Q}$ factor will quadratically reduce the fundamental linewidth at a given power level $\mathrm{P}$. The advent of the microtoroid cavity with quality factors as high as 500 million [4] and recent advances in Erbium-doped lasers using this cavity geometry [5] makes it possible to decrease the fundamental laser linewith to the $\mathrm{Hz}$ level at very modest output powers.

The laser is made from Er-doped sol-gel silica spun on a silicon substrate. The microtoroid structure is achieved by $\mathrm{CO}_{2}$ laser reflow of a sol-gel silica disk, which is fabricated using standard photolithography and buffered $\mathrm{HF}$ etching, followed by $\mathrm{XeF}_{2}$ etching techniques as described elsewhere [4,5]. The ultra-high quality of the device is due to the quick melting procedure during the $\mathrm{CO}_{2}$ reflow process and subsequent solidification into a toroidal shape due to surface tension. Optical pump power is coupled into the cavity with an efficient fiber taper [6,7] and output laser power is coupled using the same structure. Lasers were selected so as to exhibit simultaneous oscillation on "backscatter-split" optical modes. Backscatter splitting is common in Ultra-high-Q cavities [8], and, occasionally, we have found that lasers based on these cavities will simultaneously oscillate on split lines. This effect provides a convenient way to perform a heterodynemeasurement of phase noise since the common-mode "technical" noise is rejected as the modes share the same cavity.

A schematic of the experimental setup is presented in fig. 1. A tunable laser in the $1460 \mathrm{~nm}$ band is used to pump the microtoroid laser. The single-frequency pump laser is isolated using an optical circulator to prevent feedback-induced instabilities. After passing through the polarization controller, the pump wave is coupled into the microtoroid cavity by the fiber-taper. By carefully adjusting the taper-toroid coupling position, two independent lasing modes at optical frequency separation of around $10 \mathrm{MHz}$ are generated and coupled out of the cavity to the taper output end. They are subsequently divided by a 50/50 coupler. The lasing modes and the residual pump laser are monitored by an optical spectrum analyzer. The two lasing modes are further separated from the pump laser by a wavelength de-multiplexer, and detected by a photoreceiver to create a heterodyne beat note. The electrical beat-note signal is then split into two arms, which, after passing through different delay lines, recombine in an RF mixer that is connected into a spectrum analyzer.

The laser linewidth is derived from the phase-noise spectral density observed on the spectrum analyzer. Figure 2a shows the laser spectrum from the microtoroid detected by an Optical Spectrum Analyzer (OSA). The finely split laser modes are not resolved in the spectrum. Other oscillation modes are suppressed by 


\section{CMR2.pdf}

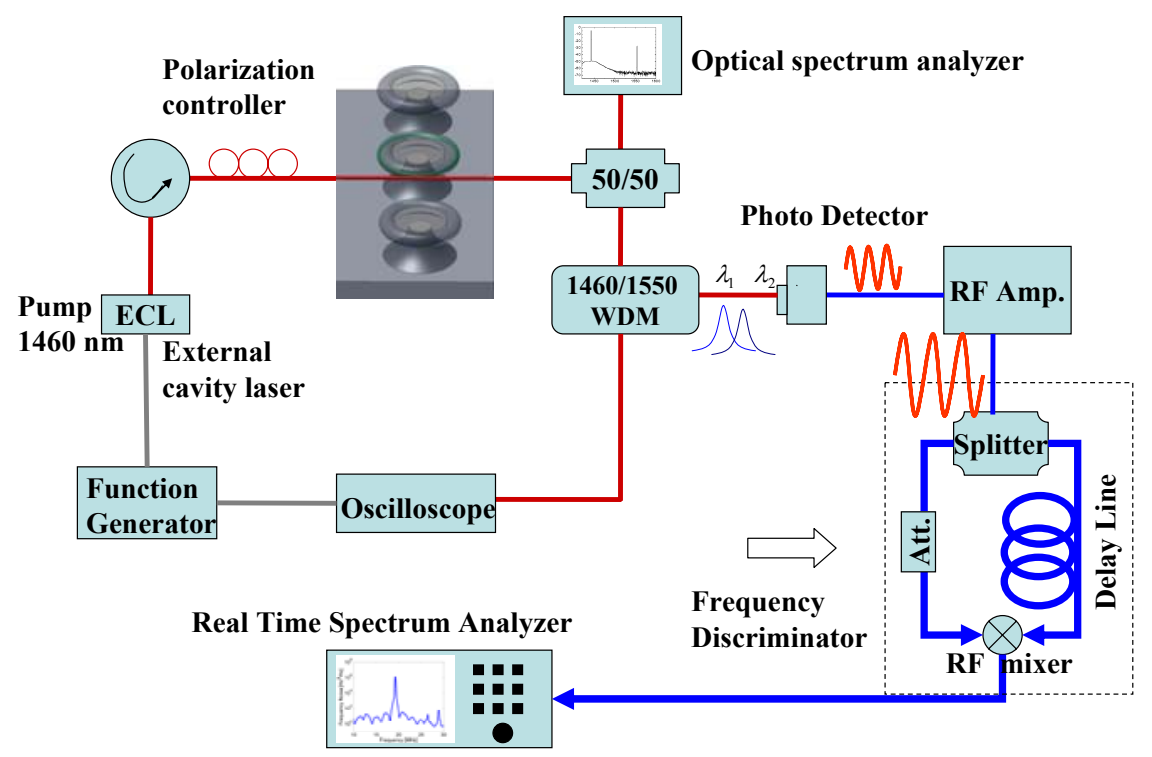

Fig 1. Schematic of the measurement setup.

more than $40 \mathrm{~dB}$. The inset of fig. 2a presents the beatnote from the two lasing modes as observed on an oscilloscope in the time domain. An optical microscope side-view image of a fiber taper coupled microtoroid is shown in the inset of fig. $2 \mathrm{~b}$. The device has a diameter of $60 \mu \mathrm{m}$ and a $\mathrm{Q}$ factor of $10^{7}$. The measured fundamental linewidth as function of inverse laser power is shown in fig. $2 \mathrm{~b}$. It clearly exhibits the classic, inverse-power dependence expected for the Schalow-Townes linewidth. At the output power of 10 microWatt, the fundamental linewidth decreases to $4 \mathrm{~Hz}$. The measured linewidth is also in close agreement with the theoretically expected value at this $\mathrm{Q}$ factor and output power level. This work was sponsored by DARPA.

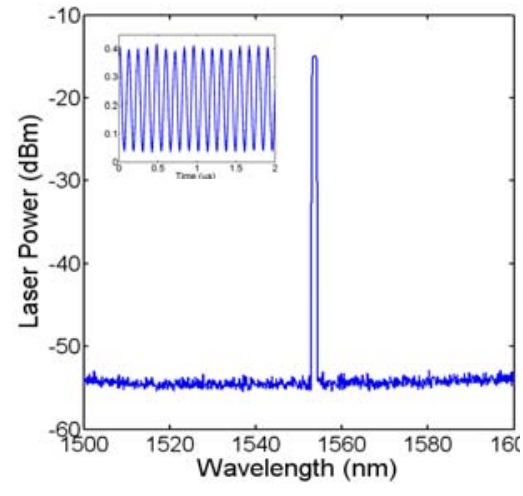

(a)

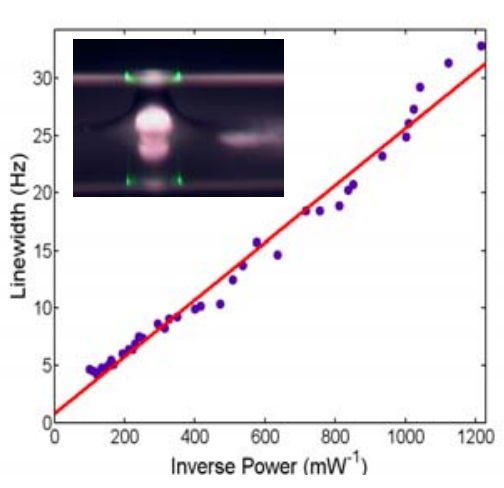

(b)

Fig 2. a. Laser spectrum . b. Linewidth plotted versus inverse laser power

[1] J. Faist, F. Capasso, D.L. Sivco, C. Sirtori, A.L. Hutchinson and A.Y. Cho, “Quantum cascade Laser," Science, 264, 553-556 (1994).

[2] T.L. Myers, R..M.Williams, M.S.Taubman, C.Gmachl, F.Capasso, D.L.Sivco, J.N.Baillargeon and A.Y.Cho, "Free-running frequency stability of mid-infrared quantum cascaded lasers,” Opt. Lett. 27, 170-172 (2002).

[3]J Geng, C. Spiegelberg and S Jiang, "Narrow linewidgth fiber laser for 100-km optical frequency domain reflectometry," IEEE Photonics Tech Lett. 17, 1827-1829 (2005).

[4]D. K. Armani, T. J. Kippenberg, S. M. Spillane et al., "Ultra-high-Q toroid microcavity on a chip," Nature 421, $925-928$ (2003).

[5] L. Yang, T. Carmon, B.K. Min, S.M. Spillane, and K. J. Vahala, "Erbium-doped and Raman microlasers on a silicon chip fabricated by the sol-gel process," Appl. Phys. Lett. 86, 091114 (2005).

[7] M. Cai, O. Painter, and K J Vahala, "Observation of critical coupling in a fiber taper to a silica microsphere whispering gallery mode system," Phy. Rev. Lett. 85, 74-77 (2000).

[8] S.M. Spillane, T. J. Kippenberg, O. J. Painter, and K. J. Vahala, "Ideality in a fiber-taper-coupled microresonator system for application to cavity quantum electrodynamics," Phy. Rev. Lett. 91, 043902 (2003).

[9] T.J. Kippenberg, S.M.Spillane, and K. J. Vahala "Modal coupling in traveling-wave resonators," Opt. Lett. 27, 1669 (2002) 